\title{
A Satisfação do Usuário em Hospital Universitário*
}

\author{
Antonio C. Lemme**, Gerson Noronha**, José B. Resende*t
}

\begin{abstract}
LEMME, A.C. et al. A satisfação do usuário em hospital universitário. Rev. Saúde públ., S. Paulo, 25: 41-6, 1991. São apresentados resultados de uma pesquisa exploratória da satisfação/insatisfação de 158 pacientes que recorreram a um Hospital Universitário (HUPE) para consulta inicial. As entrevistas foram feitas por alunos do primeiro ano, como parte de projeto de inserção precoce do mesmo com a prática médica, acompanhando pacientes como observadores. Foi utilizado questionário que cobriu os vários aspectos do problema: variáveis sócio-demográficas, arte do cuidado, resolutividade da consulta médica, sugestões de melhoria dos serviços. Tanto alunos/ observadores como os usuários conseguiram perceber com precisão os problemas do processo do atendimento (recepção, matrícula, consulta, enfermagem, serviço social, farmácia, exames complementares) mas houve divergência de opinião entre vários aspectos da satisfação/insatisfação entre alunos e usuários, provavelmente devido às origens sociais diferentes e expectativas diversas.
\end{abstract}

Descritores: Satisfação do paciente. Hospitais universitários. Estudos de avaliação.

\section{Introduçáo}

Há mais de uma década vem sendo proposta modificaçōes no sistema de avaliação dos serviços de saúde (Blum) ${ }^{4}, 1974$, tanto em nível conceitual de qualidade do atendimento (Donabedian ${ }^{9}, 1980$ ), quanto do processo e dos resultados (Lohr ${ }^{11}$, 1988). Questiona-se as atuais medidas de eficácia (Bunker ${ }^{6}, 1988$; US Congress. Office of Technology Assessment ${ }^{14}$, 1988) e, ao final dos anos 80 , é cogitada a utilização da idéia da melhoria contínua (o kaizen dos japoneses) nos serviços de saúde (Berwick ${ }^{2}, 1989$ ). Dentro da visão de controle de qualidade no atendimento médico ("vinho novo em garrafas antigas") aponta-se a medida da satisfação do usuário, como um dos fatores que devem ser analisados (Scally e col. ${ }^{18}$, 1988).

A experiência internacional é controversa quanto a utilização da ótica do usuário na avaliação dos serviços de saúde. Ressalta-se a complexidade técnica das intervençōes desses serviços, admitindose este julgamento apenas quanto ao aspecto da relação interpessoal (Cleary e Mc Neil ${ }^{7}, 1988$ ). O estudo da satisfação do usuário tem ganho destaque na literatura, à medida em que se estabelece relações entre ela e certas características dos pacientes. Assim podemos admitir que a decisão de

* Trabalho apresentado no 2 Congresso Brasileiro de Saúde Coletiva (ABRASCO) (SP), no XXVII Congresso da Associação Brasileira de Ensino Médico (MA) e na Rede Ida/Brasil (RJ), realizados em 1989.

* Departamento de Planejamento do Instituto de Medicina Social da Universidade do Estado do Rio de Janeiro - Rua São Francisco Xavier, 524 - 20550 - Rio de Janeiro, RJ Brasil. se utilizar um serviço de saúde representa "o elo final de uma cadeia de decisões que se inicia com a percepção pessoal de uma necessidade biológica, caminha para a avaliação da necessidade de cuidado médico para esta alteração, interrogando-se ainda o tipo de cuidado médico ideal, qual serão os seus custos, etc." (Stratmann $\left.{ }^{19}, 1975\right)$. Esta decisão associada à satisfação com o sistema de saúde levará à utilização de seus serviços. Mais recentemente os trabalhos tentam introduzir modelos explicativos das relações entre características sóciodemográficas, familiares e as dimensōes da satisfação (Zastony e col. ${ }^{26}, 1989$ ).

Assim, ao se definir satisfação como "uma variável causal que faz com que as pessoas procurem o atendimento médico e um resultado baseado em experiências anteriores" (Pascoe $\left.{ }^{13}, 1983\right)$, estamos apontando os dois aspectos que devem ser considerados quando da avaliação da satisfação. $\mathrm{O}$ primeiro diz respeito à satisfação com o sistema de saúde em geral o que motivará a procura de uma unidade (garantia de utilização); no segundo aspecto mede-se a satisfação com o processo do atendimento ou só com o resultado (garantia de retomo) que leva à continuidade do tratamento podendo inclusive interferir na eficácia da terapêutica pela maior adesão da mesma (Wartman e col. ${ }^{24}, 1983$ ).

Quanto à relação entre o grau de satisfação e características pessoais do usuário ainda persiste grande divergência entre os autores. No estudo realizado por Weiss 25 (1988), após uma revisão extensa sobre o tema, são apontados 4 grupos principais de determinantes da satisfação:

$1^{2}$ - Características dos pacientes, incluindo as sócio-demográficas, as expectativas sobre a 
consulta médica e o estado atual de saúde.

$2^{2}$ - Características dos profissionais que prestam 0 atendimento incluindo traços de personalidade, qualidade técnica e a "arte do cuidado" prestado.

$3^{2}$ - Aspectos da relação médico-paciente, incluindo o estilo de comunicação entre os dois, bem como o "resultado" do encontro.

$4^{9}$ - Fatores estruturais e ambientais, incluindo o acesso, forma de pagamento, tempo de tratamento, marcação de consultas e outros.

No primeiro grupo a medida da satisfação com o processo ou o resultado do atendimento apresenta muita contradição entre os autores, quanto à sua relação com as características sócio-demográficas. Admite-se ser maior a influência destas características sobre aspectos particulares do atendimento, como por exemplo - 0 acesso (Penchansky e Thomas ${ }^{17}, 1981$ ). Ainda segundo Weiss ${ }^{25}$ existiriam fatores predisponentes, individuais, que teriam ação mais definida sobre o grau de satisfação, destacando-se entre eles:

- Satisfação com a vida em geral e com a comunidade onde reside.

- Nível de credibilidade nos médicos da região.

- Sentimentos elevados de controle interno (quando o indivíduo considera-se responsável pela sua própria vida) e não de controle externo (quando imagina que a vida é determinada por fatores externos à sua pessoa).

- Continuidade no tratamento garantida pela existência de oferta regular de cuidados e o atendimento sempre feito pelo mesmo profissional.

- Estado atual da saúde que poderia interferir não só pela gravidade do quadro apresentado que influenciaria de modo positivo ou negativo, mas também pela limitação física, psicológica, social ou mental decorrente da doença. Nesta situação foi constatado que a satisfação está fortemente relacionada com o quanto de informação o paciente recebe por parte do profissional de saúde sobre sua patologia (Patrick e col. ${ }^{15}$, 1983).

Ainda no primeiro grupo deve-se destacar que as expectativas sobre a consulta médica e os desejos dos pacientes devem ser melhor explorados pelos médicos pois os usuários têm dificuldade de os expressar verbalmente e, ao não terem respostas, estes sentimentos poderão transformar-se em fator de tensão e frustração (Uhlman e col. ${ }^{21}$, 1984).

No segundo e terceiro grupos de determinantes (características dos profissionais e aspectos da relação médico-paciente) deve-se considerar que a atitude passiva do paciente frente ao saber médico e a dificuldade de expressar verbalmente suas dúvidas e inquietações somam-se à natureza despersonalizada da moderna tecnologia médica, dificultando ao médico avaliar a satis- fação do usuário no transcorrer de uma consulta (Merkel 12, 1984). Na comunicação entre os médicos e os pacientes está certamente um dos mais importantes aspectos da satisfação. Sabe-se, atualmente, que o estilo da comunicação predomina sobre a qualidade técnica no transcorrer da consulta, principalmente após já ter havido a escolha ou a indicação do médico (Buller e Buller ${ }^{5}$, 1987). O estilo de comunicação entre o usuário e - profissional de saúde pode ser afetivo ("affiliation") ou de "controle" ("control"). Na primeira situação o médico mostra interesse, amizade, desejo de ajudar, simpatia, humor e calor humano, o que não acontece no outro estilo. As melhores respostas ao tratamento e o maior grau de satisfação estão significativamente relacionados com o primeiro estilo.

No quarto grupo de determinantes foi demonstrada a importância dos fatores estruturais e ambientais que afetam de maneira indireta o padrão de utilização e o acesso. Assim, por exemplo, homens brancos de nivel educacional superior valorizam 0 horário do atendimento e o sistema de marcação ("walk-in-facilities"). Já as donas de casas e os mais jovens têm sua preocupação voltada principalmente para os custos da assistência médica e a capacidade de pagá-los (Thomas e Penchansky ${ }^{20}$, 1984).

A escassez de artigos publicados no Brasil e mesmo na América Latina (Pedersen ${ }^{16}, 1987$ ) sobre o tema garantia de qualidade - satisfação do usuário, estimulou a Equipe Coordenadora desta pesquisa no sentido de suprir esta lacuna. Pretendeu-se, também, agir "diminuindo a distância entre os elegantes estudos e métodos de pesquisa e o dia a dia dos administradores", objetivo considerado fundamental, na década de 90 , para o avanço dos trabalhos experimentais desenvolvidos nos serviços de saúde (Berwick ${ }^{3}, 1989$ ).

Por outro lado a revisão efetuada na bibliografia internacional mostra que o padrão sócioeconômico e cultural da população que se utiliza dos serviços públicos de saúde locais é bem diferente dos paises onde foram feitos os trabalhos anteriormente citados. Por esta razão, optou-se por iniciar com um estudo exploratório, a fim de colher dados e confeccionar instrumentais adequados à nossa realidade.

O presente estudo visa, portanto, a avaliar qualitativamente a assistência prestada por uma unidade de saúde onde poderiam ser pesquisados alguns dos fatores determinantes e predisponentes da satisfação/insatisfação do usuário propostos por Weiss 25 e alguns outros pertinentes às características sócio-culturais do país, e cujos objetivos da unidade de saúde sejam a universalidade, a equidade e a resolutividade. 


\section{Material e Método}

A unidade de saúde escolhida foi um hospital universitário do Município do Rio de Janeiro (HU).

$O$ instrumental elaborado pela equipe de Coordenação da pesquisa foi aplicado por alunos do primeiro ano de faculdade de ciências médicas do mesmo Município. Estes foram previamente orientados a se apresentarem sem sinais (uniforme e/ou crachá) que permitissem sua identificação por parte dos integrantes da estrutura hospitalar, o que poderia modificar o comportamento habitual dos profissionais na relação com os usuários.

$O$ aluno apresentava-se a um paciente de primeira consulta e, após obter o seu consentimento, acompanhava-o desde a sua chegada à porta da frente do hospital até a sua retirada da instituição, sem ajudá-lo ou influenciar nos obstáculos porventura encontrados durante este percurso. Após esta experiência, $\mathrm{o}$ aluno preenchia o questionário com o consultante, quando então eram anotados dados de identificação, condiçōes sócio-econômicas e feita uma avaliação de qualidade do atendimento, incluindo o relacionamento interpessoal do paciente com os diversos profissionais da equipe de saúde, como também a organização e o funcionamento de dois setores intermediários (informação e matrícula). Solicitava-se, ainda, a opinião do ponto de vista do usuário, sobre a resolutividade da consulta, assim como sobre o fornecimento de receituário e de medicamentos. Por último, indagava-se a impressão sobre a instituição hospitalar e solicitava-se a indicação dos grupamentos aos quais o paciente recorreria, caso fosse necessário, para reivindicar seus direitos e apresentar queixas quanto ao atendimento recebido.

\section{Resultados}

$\mathrm{Na}$ análise dos dados sobre a satisfação do usuário foram considerados apenas 158 questionários, do total de 260 aplicados. Foram desprezados os que não estavam corretamente preenchidos ou que não haviam explorado os itens referentes ao tema.

Ressaltamos, inicialmente, que o tempo de permanência no $H U$, desde a entrada até a obtenção da consulta desejada, levou entre 3 a 4 horas, o que foi motivo de freqüentes reclamações e desentendimentos.

Embora 75\% dos pacientes tenham sido atendidos no mesmo dia, destacamos o fato de que $47 \%$ o foram no Serviço de Pronto Atendimento (SPA), $16 \%$ na Clínica Médica e $16 \%$ na Pediatria. Os atendimentos em ambulatórios especializados atingiram apenas $13 \%$, sendo que a cirurgia, a ginecologia e a
Tabela 1. Serviço do hospital que prestou atendimento de primeira vez

\begin{tabular}{lcc}
\hline Serviço* & Freqüência & $\%$ \\
\hline Pronto atendimento & 74 & 46,8 \\
Clínica médica & 25 & 15,8 \\
Pediatria & 25 & 15,8 \\
Outras especialidades ${ }^{\star *}$ & 20 & 12,7 \\
Ginecologia & 7 & 4,4 \\
Psiquiatria & 2 & 1,3 \\
Cirurgia Geral & 1 & 0,6 \\
\hline
\end{tabular}

- Dado nâo assinalado em quatro questionários

** Cardiologia, Neurologia e outras.

Tabela 2. Satisfaçâo da clientela com os serviços utilizados e com o hospital em geral

\begin{tabular}{|c|c|c|c|c|c|}
\hline \multirow{3}{*}{ Serviços* } & \multicolumn{5}{|c|}{ Atendimento } \\
\hline & \multicolumn{2}{|c|}{ Satisfatório } & \multicolumn{2}{|c|}{ Insatisfatório } & \multirow{2}{*}{$\begin{array}{c}\text { Total } \\
\text { pacientes }\end{array}$} \\
\hline & $n^{2}$ & $\%$ & $n^{2}$ & $\%$ & \\
\hline Informação & 89 & 66 & 45 & 34 & 134 \\
\hline Matricula & 84 & 63 & 50 & 37 & 134 \\
\hline A. Social & 24 & 70,5 & 10 & 29,5 & 34 \\
\hline Enfermagem & 33 & 82,5 & & 17,5 & \\
\hline MÉdicos & 136 & 90 & 15 & 10 & 151 \\
\hline Hospital"* & 106 & 71 & 43 & 29 & 149 \\
\hline
\end{tabular}

- Os pacientes podem ter se utilizado de mais de um serviço.

* Năo opinaram nove pacientes

psiquiatria não alcançaram 5\% (Tabela 1). Este último serviço possui outra porta de entrada, nos fundos do HU, habitualmente utilizada pelos pacientes.

Quanto à categoria profissional envolvida no atendimento os médicos e médicos-residentes representaram $88 \%$, o Serviço Social $2,5 \%$ e a Enfermagem $1,3 \%$, estes dois serviços quando únicos a prestar a consulta.

Dos 158 consultantes apenas $61 \%$ recebeu uma receita. Entre aqueles que tinham uma receita (97) e recorreram à farmácia apenas $32(34 \%)$ foram atendidos.

Destacamos, também, a freqüência com que os pacientes (69) retornavam para exames complementares $(43,7 \%)$ e houve a necessidade de uma segunda consulta para 106 pacientes (67\%). Entre os entrevistados 72 (45\%) consideraram que o seu problema de saúde fora resolvido. Por outro lado, $51(32 \%)$ declararam que não havia sido alterado e 35 (23\%) não opinaram.

Quanto à satisfação da clientela com os diversos serviços do HU e com o hospital como instituição, o setor de matrícula foi o que apresentou menor grau de satisfação (63\%). Foram considerados os mais satisfatórios o atendimento médico (90\%) e o serviço de enfermagem $(82,5 \%)$ (Tabela 2$)$. 
Para 102 participantes desta pesquisa, é a direção do HU $(64,6 \%)$ quem deve receber suas reclamações. Foram citados ainda o Serviço Social (19\%), a Imprensa $(17,1 \%)$ e Associação de Moradores $(10,8 \%)$ como possiveis canais de reclamação.

\section{Discussão}

Todo o trabalho teve como núcleo central de pesquisa a investigação do funcionamento de um hospital universitário na ótica do usuário e o seu grau de satisfação com os serviços prestados. Ao se utilizar como pesquisadores alunos da graduação imaginou-se que eles poderão interferir, futuramente, como médicos ou planejadores, na qualidade do atendimento no setor público de saúde.

$\mathrm{Na}$ amostra trabalhada não foi possível, devido à extrema homogeneidade dos usuários, estabelecer correlações fortes entre variáveis sóciodemográficas e o grau de satisfação. Quanto a renda, a população-alvo situou-se numa faixa muito estreita de até dois salários mínimos. Notou-se, porém, a exclusão das classes de melhor nível de renda e que habitualmente são as que reivindicam melhores condições de atendimento (Vianna ${ }^{22}$, 1987). Tais cidadãos já não recorrem ao HU nem mesmo para a realização de procedimentos de tecnologia mais sofisticada. O mesmo aconteceu com a escolaridade, onde poucos ultrapassaram o primeiro grau. A predominância das mulheres como consultantes e acompanhantes de crianças, como também o pequeno número de jovens e idosos não permitiu estabelecimento de relações fortes entre sexo, idade e o grau de satisfação.

A demanda, preponderantemente espontânea, com patologias de pouca complexidade e a inexistência de mecanismos de contra-referência com absorção inadequada de clientela, vem transformando o perfil do hospital. Gera-se, assim, um racionamento na oferta de consultas iniciais provocando insatisfação na porta de entrada $e$ cristalizando na população motivos para a não utilização da unidade.

A hegemonia dentro do aparelho simbólico do usuário de um padrão de assistência centrada na consulta médica é reforçada quando se defronta com uma instituição prestadora de serviços, que apesar de possuir uma gama considerável de profissionais (enfermagem, serviço social, nutrição) não os utiliza, perpetuando o conceito de atendimento médico e não de atendimento à saúde. Esta desvalia dos outros profissionais como prestadores de consulta, transforma-se num mecanismo de barreira do acesso gerando insatisfação. Outra grande limitação ao acesso está representada pelo grau de insatisfação com os setores de informação e matrícula que ainda não incorporaram na sua prática avanços tanto da informática, quanto de uma programação visual dentro do hospital.

Uma contradição foi observada entre a percepção do aluno/observador e o entrevistado. $O$ aluno, por ser de nível sócio-econômico mais elevado e, portanto, com maiores expectativas e exigências, demonstrou inúmeras reservas e críticas (bastante contundentes) à qualidade do atendimento nos seus diversos niveis, saindo do hospital profundamente insatisfeito com o que viu e sem entender 0 alto grau de satisfação do usuário.

O caráter episódico e emergencial do atendimento no SPA não permitiu a avaliação da qualidade técnica do serviço prestado, sendo mais destacado pelos pacientes o aspecto do relacionamento interpessoal e a resolutividade da consulta. No relacionamento médico-paciente houve alguma discordância entre os quesitos tipo "score": (ruim, regular, bom e ótimo) e as respostas às perguntas em aberto. Embora um alto índice de satisfação com os médicos nas perguntas de "score", foram frequentes as queixas de desatenção, desinteresse, falta de calor humano, impontualidade e o tempo exíguo dedicado à consulta, quando o paciente respondia livremente às perguntas abertas. Entre as especialidades médicas confirmou-se a alta satisfação com os pediatras, notórios pelo estilo de comunicação. $O$ elevado número de informaçōes que fornecem às mães as faz ficar muito satisfeitas não só com a consulta, mas também com a unidade onde se dá o atendimento (Becker e col. 1, 1972; Deisher e col. ${ }^{8}, 1965$ ).

Obstáculo observado, com graves implicaçðes na qualidade e resolutividade do atendimento, diz respeito a insuficiente oferta de medicamentos à clientela do $\mathrm{HU}$, que por sua condição sócioeconômica não tem condições de arcar com os custos de compra. A longo prazo, o não fornecimento dos remédios receitados vai provocar uma "insatisfação tardia" o que levará o paciente a considerar o seu problema não resolvido e o incentivará a procurar outra unidade.

Mesmo não sendo o objetivo imediato da pesquisa e certamente impossivel de ser medido numa consulta no SPA, supomos que o cumprimento das prescriçôes, a continuidade do tratamento e a conseqüente eficácia da terapêutica fiquem muito prejudicados diante da situação descrita. $\mathrm{O}$ aumento da insatisfação com os serviços públicos de saúde leva o usuário não ao "doctor shop" relatado em pesquisas americanas (Kasteler e col. 10, 1976), mas cria um personagem que perambula, de porta em porta das nossas unidades, a procura de um direito, consagrado constitucionalmente, mas ainda não compreendido por alguns profissionais de saúde, ou pela instituição como um todo. 
Como último destaque, anotamos a importância que a população dá à direção do hospital como locus de poder e credibilidade para terem os seus problemas resolvidos. Além da direção, outros segmentos, ainda que minoritários, foram apontados pela clientela demonstrando uma consciência participativa incipiente.

\section{Conciusóes}

A utilização da satisfação da clientela na avaliação da qualidade do serviço prestado, tema sobre o qual existe uma ampla literatura internacional (OTA 14,1988), necessita de um aprofundamento em nosso meio, já que a literatura brasileira é praticamente ausente nesse debate.

No presente estudo exploratório com usuários do $\mathrm{HU}$, de baixa renda e com pouca escolaridade, ficou mostrada a sua capacidade de, ao expressarem a sua satisfação/insatisfação com os diversos setores e serviços do hospital, identificarem a origem dos obstáculos que impedem um atendimento de melhor qualidade.

A partir desta pesquisa consideramos necessário fazer algumas modificações nos futuros trabalhos, quanto ao instrumental de avaliação e aos locais de aplicação. No primeiro aspecto destacaríamos que a diferença, entre a realidade de outros países e o padrão sócio-econômico e cultural da população que freqüenta os nossos serviços públicos, exige uma adaptação/transformação dos instrumentais usados naqueles países. Esta modificação abrangeria não só a linguagem e a utilização de conceitos inexistentes em nossa cultura, mas especialmente o que se refere à investigação dos custos, financiamento e dados ambientais,aspectos de relevo nos países onde a saúde é considerada um bem de consumo individual e não um direito de uma população universalmente coberta pelo Estado. É necessário também atentar para o fato de que já foi comprovada uma tendência por parte dos usuários de classes sociais menos favorecidas de avaliar positivamente os serviços que the são prestados (Ware ${ }^{23}, 1978$ e OTA ${ }^{14}, 1988$ ). Como foi anteriormente comentado houve uma discrepância muito acentuada entre a opinião dos alunosobservadores e a dos usuários acompanhados. Para diminuir este viés, pretendemos modificar o questionário, ampliando as perguntas do estilo SIM/ $\mathrm{NÃO}$, procurando misturar aspectos positivos e negativos do atendimento, conforme sugestão da OTA (OTA ${ }^{14}, 1988$ ) e explorar com um maior número de perguntas em aberto as expectativas e desejos dos pacientes.

Quanto ao local de aplicação pretendemos utilizar outros cenários para ampliar os dados e futura- mente elaborarmos, se possivel, um instrumental único para aplicação no sistema público de saúde. Acreditamos que algumas entrevistas devam ser realizadas na residência do consultante, após o atendimento, onde ele certamente se sentiria mais a vontade para responder às perguntas referentes aos serviços prestados. Em outra modalidade de pesquisa a avaliação da satisfação seria feita na residência do usuário, sorteado aleatoriamente, e que daria o seu depoimento sobre os serviços de saúde que habitualmente utiliza. Pretende-se com esta atitude ultrapassar o viés das pesquisas executadas na porta de unidades, nas quais ignora-se toda uma população que não se serve da unidade, inclusive por estar insatisfeita com o serviço de saúde como um todo.

LEMME, A.C. et al. [User satisfaction at a university hospital]. Rev Saúde públ., S.Paulo, 25: 41-6, 1991. The results of a research project into the level of satisfaction/dissatisfaction of 158 first-time patients attending by an University hospital, Brazil, are presented. The interviews were carried out by first year medical students as part of a comprehensive project concerned with the restructuring of the Medical Curriculum. Its prime objective was to expose the students to patients/ consumers as early as possible in their studies. After an introductory training program, the students asked the patients arriving at the hospital out-patient clinic for permission to observe them throughout the attendance given. A questionnaire was used which covered the various aspects of the consumers relationship with a medical institution (socio-economic variables, "art of care", outcome of the medical encounter and suggestions for improvement). Both patients and students were able to perceive the different problems that patients confront in the course of their dealings with a medical institution (reception, registration, nursing, social service and medical attention). The data showed a difference of opinion between students and patients regarding several aspects of the satisfaction/dissatisfaction items, partly because of their different social class status and parthy due to the different expectations regarding medical care.

Keywords: Consumer satisfaction. Hospitals, university. Evaluation studies.

\section{Referênclas Bibliográficas}

1. BECKER, M.H.; DRACHMAN, R.H.; KIRSCHT, J.P. Predicting mothers compliance with Pediatric Medical Regimens. J. Pediat., 81: 843-54, 1972.

2. BERWICK, M.D. Continuous improvement as an ideal in health care. New Engl. J. Med, 320: 53-6, 1989.

3. BERWICK, M.D. Health services research and quality of care assigments for the 1990s. Med.Care, 27: 763-71, 1989.

4. BLUM, L.H. Evaluating health care. Med.Care, 12: 999 $1011,1974$.

5. BULLER, K.M. \& BULLER, B.D. Physicians communication style and patient satisfaction. J Hlth soc. Behav., 28: 375-88, 1987.

6. BUNKER, P.J. Is efficacy the gold standart of quality as- 
sessmen? Inquiry, 25: 51-8, 1988.

7. CLEARY, D.P. \& McNEIL, J.B. Patient satisfaction as an indicator of quality care. Inquiry, 25: 25-36, 1988.

8. DEISHER, R.W.; ENGEL, R.L.; SPIELHOZ, R.; STANDFAST, J.I. Mothers' opinion of their pediatric care. Pediatrics, 35: 82-90, 1965.

9. DONABEDIAN, A. Exploration in quality assessment and monitoring. The definition of quality and approaches to its assessment. Ann Arbor, Mich., Health Administration Press, 1980. v.1. p. 1-31.

10. KASTELER, J.; KANE, R.L.; OLOSEN, D.M.; THETFORD, C. Issues underlyng prevalence of "DoctorShopping" behavior. J.Hlth soc. Behav., 17: 328-39, 1976.

11. LOHR, N.K. Outcome measurements: concepts and questions. Inquiry, 25: 37-50, 1988.

12. MERKEL, T.W. Physician perception of patient satisfaction: do doctor know which patients are satisfied? Med.Care, 22: 453-9, 1984.

13. PASCOE, G.C. Patient satisfaction in primary health care: a literature review and analysis. Eval. Progr. Plann., 6: 185-210, 1983.

14. PATIENTS assessment of their care. In: US Congress. Office of Technology Assessment. The quality of medical care: information for consumers. Washington, D.C., U.S. Government Printing Office, 1988. p. $231-47$

15. PATRICK, L.D.; SCRIVENS, E.; CHARLTON, R.H.J. Disability and patient satisfaction with medical care. Med.Care, 21: 1063-75, 1983.

16. PEDERSEN, D. Evaluación de servicios de salud: la perspectiva de la gente. Caraballeda, Venezuela, Organización Panamericana de la Salud/Ministerio de Salud de Venezuela, 1987. p. 12-6.

17. PENCHANSKY, R. \& THOMAS, W.J. The concept of access: definition and relationship to consumer satisfaction. Med. Care, 19: 127-41, 1981.
18. SCALLY, G.; WILDE, J.; CORRIGAN, D. Quality assurance: new wine in old bottles. Publ. Hlth, 102: 5814, 1988.

19. STRATMANN, C.W. A study of consumer attitudes about health care: the delivery of ambulatory services. Med.Care, 13: 537-48, 1975.

20. THOMAS, J.W. \& PENCHANSKY, R. Relating satisfaction with access to utilization of services. Med.Care, 22: 553-68, 1984.

21. UHLMANN, F.R.; INUI, S.T.; CARTER, B.W. Patient requests and expectations: definitions and clinical applications. Med.Care, 22: 681-5, 1984.

22. VIANNA, M.L.T. W., coord. O INAMPS enquanto organizaçâo e suas clientelas. In: Fiori, J.L., coord. geral. Diagnóstico de alguns obstáculos a uma ação planejada do INAMPS; relatório final. s.1., IEI-UFRJ/ INAMPS/FINEP, 1987. v. 1. [Mimeografado].

23. WARE, J.E. Effects of aquiescent response set on patient satisfaction ratings. Med.Care, 16:327-36, 1978.

24. WARTMAN, A.S.; MORLOCK, L.L.; MALITZ, F.E.; PALM, A.E. Patient understanding and satisfaction as predictors of compliance. Med.Care, 21: 386-91, 1983.

25. WEISS, L.G. Patient satisfaction with primary medical care: evaluation of sociodemographic and predispositional factors. Med.Care, 26: 383-92, 1988.

26. ZASTOWNY, R.T.; ROGHMANN, J.K.; CAFFERATA, L.G. Patient satisfaction and the use of health services: explorations in causality. Med.Care, 27: 705-23, 1989.

Recebido para publicação em 11/5/1990. Reapresentado em 2111111990. Aprovado para publicação em 29/11/1990. 